\title{
Routing Strategy for LEO Satellite Networks Based on Membership Degree Functions
}

\author{
Jian Zhou $\mathbb{D}^{1,2}$ Qian Bo, ${ }^{1,2}$ Lijuan Sun $\mathbb{D}^{1,2}$ Juan Wang, ${ }^{1,2}$ and Xiaoyong Yan ${ }^{1,2}$ \\ ${ }^{1}$ College of Computer, Nanjing University of Posts and Telecommunications, Nanjing 210023, China \\ ${ }^{2}$ Jiangsu High Technology Research Key Laboratory for Wireless Sensor Networks, \\ Nanjing University of Posts and Telecommunications, Nanjing 210023, China \\ Correspondence should be addressed to Jian Zhou; zhoujian@njupt.edu.cn and Lijuan Sun; lucifinil919@126.com
}

Received 3 August 2020; Revised 10 September 2020; Accepted 5 October 2020; Published 16 October 2020

Academic Editor: Xiaolong Xu

Copyright (C) 2020 Jian Zhou et al. This is an open access article distributed under the Creative Commons Attribution License, which permits unrestricted use, distribution, and reproduction in any medium, provided the original work is properly cited.

The deployment of Mobile Edge Computing (MEC) servers on Low Earth Orbit (LEO) satellites to form MEC satellites is of increasing concern. A routing strategy is the key technology in MEC satellites. To solve the uncertainty problem of LEO satellite link information caused by complex space environments, a routing strategy for LEO satellite networks based on membership degree functions is proposed. First, a routing model based on uncertain link information is established. In particular, the membership function is designed to describe the uncertain link information. Based on this, the comprehensive evaluation of the path is calculated, and the routing model considering uncertainty is established with the comprehensive evaluation of the path as the optimization objective. Second, in order to quickly calculate the path, a grey wolf optimization algorithm is designed to solve the routing model. Finally, simulation results show that the proposed strategy can achieve efficient and secure routing in complex space environments and improve the overall performance compared with the performances of traditional routing strategies.

\section{Introduction}

Mobile Edge Computing (MEC), which is a novel and powerful paradigm, is a promising alternative for providing computing capabilities at the edges of networks [1-3]. Low Earth Orbit (LEO) satellite networks possess the advantages of near real-time, wide coverage, and anti-destructive properties $[4,5]$. Recently, some studies have combined LEO satellite networks with MEC to deploy MEC servers on LEO satellites for reducing delays and more general purposes [6-8]. As a new MEC architecture, MEC satellites are an emerging topic [9-11]. The design of communication protocols in MEC satellites for achieving efficient and secure data transmission is currently a research hotspot.

As the core of the communication protocol, the routing strategy for satellite networks is responsible for data transmission between the intersatellite links and for determining the overall performance of satellite networks [12]. Compared with terrestrial networks, satellite networks possess dynamic topological structures and unbalanced data traffic, which makes the routing strategy for terrestrial networks unsuitable for satellite networks. Thus, the routing strategy for satellite networks has been specially researched [13]. The existing research has focused on the high dynamic change issue of satellite networks. Based on the predictability, periodicity, regularity, and other features of satellite networks, routing strategies based on virtual topology [14-16], virtual nodes $[17,18]$, and coverage domain partitioning [19] were proposed. At the same time, the data traffic imbalance problem was also of high concern, and a routing strategy based on the load balance was proposed [20-22].

However, the link information of LEO satellite networks has a certain degree of uncertainty. On the one hand, the complexity of LEO satellite networks and operating space environments leads to uncertainty in the measured values of link information [23]. For example, harsh space environments such as vacuums, solar radiation, and weak magnetic fields bring about LEO satellite faults, which result in packet loss. The high-speed movement of LEO satellites brings 
about the issue of dynamic distances between satellites, which results in the instability of the transmission delay. The relatively long delay of intersatellite links results in the nonreal-time updating of link information. On the other hand, link evaluations themselves, such as "low delay," "high reliability," and "high bandwidth," possess a certain degree of uncertainty. In summary, traditional routing strategies for satellite networks are not efficient because they do not consider the complicated factors that affect the routing process. Therefore, the uncertainty of link information cannot be ignored.

In recent years, uncertain routing strategies in complex environments have attracted the attention of researchers [24] and have been successfully applied in the field of wireless sensor networks [25, 26]. At present, some researchers have focused on uncertain routing strategies in satellite networks. Zhang et al. [27] transformed multiattribute parameters of satellites into a comprehensive parameter through the Choquet fuzzy integral. Li et al. [28] estimated the congestion status between adjacent satellites through fuzzy congestion indicators and then proposed a fuzzy routing strategy to avoid congestion. Jiang et al. [29] established a fuzzy rule set and proposed a fuzzy routing strategy that satisfies multiservice QoS for satellite networks. This paper considers the uncertainty of link information, such as the transmission delay, packet loss rate, and available bandwidth, and studies the uncertain routing strategy for LEO satellite networks.

To solve the uncertainty problem of LEO satellite link information caused by complex space environments, a routing strategy based on membership degree functions is proposed in this paper. The contributions of this paper are as follows:

(1) A routing model based on uncertain link information is established. Specifically, the uncertain link information is described by the membership degree function, and then the comprehensive evaluation of the path is obtained by integrating different link information of each link in the path. On this basis, the routing model considering uncertainty is established according to the comprehensive evaluation of the path.

(2) A grey wolf optimization (GWO) algorithm is designed to solve the routing model on the premise of ensuring the validity of the path.

(3) Simulation results are presented to show that the proposed strategy can obtain the optimal paths in complex space environments and improve the performance in terms of average delay, packet loss rate, and throughput, compared with traditional routing strategies.

The remainder of the paper is organized as follows: the background of membership degree functions is given in Section 2. The overall process of the proposed strategy is described in Section 3. The routing model based on uncertain link information is established in Section 4. The GWO algorithm is designed to solve the routing model in
Section 5. The simulation analysis is given in Section 6 . Finally, the conclusion of the paper is given in Section 7.

\section{Background of Membership Degree Functions}

With the increasing demand for the description and calculation of uncertain data, uncertainty theory continues to evolve. The membership degree function in fuzzy set theory is an efficient tool for describing and dealing with the fuzziness of data [30]. The basic idea of the membership degree function is to extend classical Boolean logic to continuous logic with arbitrary values in a certain interval [31]. Due to the uncertainty of link information, the uncertain link information is described by the membership degree function in fuzzy set theory, which can better reflect the actual situation of a LEO satellite network. The basic information regarding membership degree functions is given in this section.

Let $U$ be the domain. $\mu_{A}: U[0,1]$ is a mapping from the domain $U$ to the interval $[0,1]$, and $A$ is called a fuzzy subset of $U . \mu_{A}$ is called the membership degree function of $A$. If $x \in U$, the value of $\mu_{A}(x)$ represents the fuzzy degree to which $x$ belongs to $A$ [32].

The closer the value of $\mu_{A}(x)$ is to 1 , the higher the degree to which $x$ belongs to $A$. In contrast, the closer the value of $\mu_{A}(x)$ is to 0 , the lower the degree to which $x$ belongs to $A$. In particular, when $\mu_{A}(x)=1, x$ is considered to belong to $A$ entirely. On the other hand, when $\mu_{A}(x)=0$, $x$ is not considered to belong to $A$ at all.

In this paper, we suppose that $x \in R^{+}, U$ is the link information, and $A$ is a certain kind of link information in $U$, e.g., a low transmission delay, low packet loss rate, or high available bandwidth.

\section{Overall Process of the Proposed Strategy}

The overall process of the proposed strategy is shown in Figure 1. The specific steps are as follows: first, the virtual node method is employed to solve the dynamic change issue of the LEO satellite network, and then the LEO satellite network is represented by a directed graph. Second, the uncertain link information is described by the designed membership degree function. Third, the distance between the membership degree function of link information and the ideal point is calculated, which is employed as the comprehensive evaluation of the link, and then the comprehensive evaluation of the path is obtained by accumulating the comprehensive evaluations of all links in the path. Fourth, the comprehensive evaluation of the path is taken as the optimization objective for establishing the routing model. Finally, the routing model is solved by GWO, and the optimal path can be obtained.

\section{Routing Model Based on Uncertain Link Information}

4.1. Link Information Description. In this section, taking the transmission delay, packet loss rate, and available bandwidth as examples, the link information of LEO satellite networks 


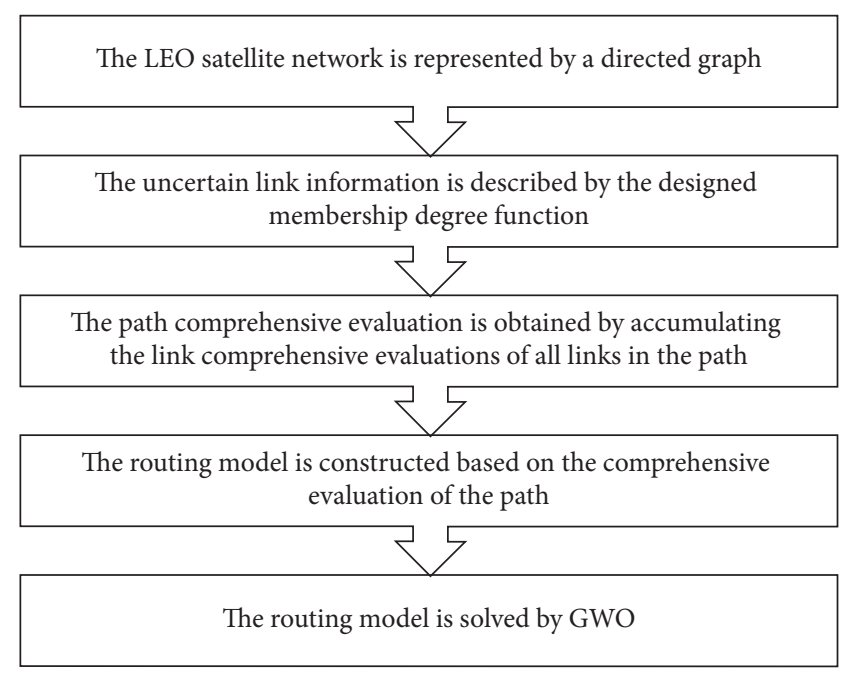

FIGURE 1: Overall process of the proposed strategy.

is represented by the designed membership degree function, which can better reflect the actual situation of a LEO satellite network. The transmission delay from satellite node $g$ to satellite node $k$ is denoted as $\operatorname{td}_{g k}$. The packet loss rate from satellite node $g$ to satellite node $k$ is denoted as $\operatorname{lr}_{g k}$. The available bandwidth from satellite node $g$ to satellite node $k$ is denoted as $\mathrm{bd}_{g k}$. In particular, $\mathrm{td}_{g k}, \mathrm{lr}_{g k}$, and $\mathrm{bd}_{g k} \in R^{+}$.

In general, the link information can be divided into efficiency indexes (the larger the better) and cost indexes (the smaller the better). To eliminate the influences of different types of link information on LEO satellite networks, the link information needs to be normalized.

\subsubsection{Membership Degree Function of the Transmission} Delay. The high-speed movement of LEO satellites brings about the issue of dynamic distances between satellites. Hence, the transmission delay is dynamically changed, which results in the uncertainty of the transmission delay. When $\mathrm{td}_{g k}$ is less than or equal to $\overline{\mathrm{Td}_{g k}}$, the transmission delay is considered to have already conformed to the optimal link, so the membership degree function of the transmission delay is invariably 1 . When $\operatorname{td}_{g k}$ is greater than $\overline{\operatorname{Td}_{g k}}$, the degree of conformation of the transmission delay to the optimal link stabilizes relatively at the beginning and then decreases rapidly. For that reason, the membership degree function of the transmission delay of link $(g, k)$ is described by the exponential function as follows:

$$
\mathrm{mf}_{g k}^{\mathrm{td}}= \begin{cases}1, & \mathrm{td}_{g k} \leq \overline{\mathrm{Td}_{g k}}, \\ e^{-\mathrm{td}_{g k}+\overline{\mathrm{Td}_{g k}},} & \mathrm{td}_{g k}>\overline{\mathrm{Td}_{g k}},\end{cases}
$$

where $\overline{\operatorname{Td}_{g k}}$ is the transmission delay threshold of link $(g, k)$. Since the transmission delay is an additive parameter, it is calculated as $\overline{\mathrm{Td}_{g k}}=\mathrm{Td}_{i j} / \mathrm{Hc}_{i j}$, where $\mathrm{Td}_{i j}$ is the maximum transmission delay threshold of the path, and $\mathrm{Hc}_{i j}$ is the maximum hop count threshold of the baseline path. $\overline{\operatorname{Td}_{g k}}$ represents the fact that the transmission delay threshold is averaged to every link in the path. The transmission delay can be denoted by $\operatorname{td}_{g k}=L_{g k} / \mathrm{vt}_{g k}$, where $L_{g k}$ is the link distance from satellite node $g$ to satellite node $k$ and $\mathrm{vt}_{g k}$ is the constant of light velocity. The smaller $\mathrm{td}_{g k}$ is, the more consistent the optimal link. When $\mathrm{td}_{g k}$ is less than or equal to $\overline{\mathrm{Td}_{g k}}, \mathrm{mf}_{g k}^{\mathrm{td}}$ is invariably 1 , which demonstrates that the current link completely conforms to the optimal link. When $\mathrm{td}_{g k}$ is greater than $\overline{\mathrm{Td}_{g k}}$, the membership degree of the transmission delay decreases as $\operatorname{td}_{g k}$ increases. That is, the degree of conformation to the optimal link decreases.

4.1.2. Membership Degree Function of the Packet Loss Rate. A harsh space environment, such as a vacuum, an area with solar radiation, or a weak magnetic field, brings about LEO satellite faults, which result in packet loss. Thus, the packet loss rate possesses a certain uncertainty. When $\operatorname{lr}_{g k}$ is less than or equal to $\overline{\operatorname{Lr}_{g k}}$, the packet loss rate is considered to have already conformed to the optimal link, so the membership degree function of the packet loss rate is invariably 1 . When $\operatorname{lr}_{g k}$ is greater than $\overline{\operatorname{Lr}_{g k}}$, the degree of conformation of the packet loss rate to the optimal link decreases linearly as the packet loss rate increases. For that reason, the membership degree function of the packet loss rate of link $(g, k)$ is described by the linear function as follows:

$$
\mathrm{mf}_{g k}^{\mathrm{lr}}= \begin{cases}1, & \mathrm{lr}_{\mathrm{gk}} \leq \overline{\mathrm{Lr}_{g k}}, \\ \frac{1-\operatorname{lr}_{g k}}{1-\overline{\operatorname{Lr}_{g k}}}, & \operatorname{lr_{gk}}>\overline{\operatorname{Lr}_{g k}},\end{cases}
$$

where $\overline{\operatorname{Lr}_{g k}}$ is the packet loss rate threshold of link $(g, k)$. Since the packet loss rate is a multiplicative parameter, it is calculated as $\overline{\operatorname{Lr}_{g k}}=\sqrt[H c i j]{\operatorname{Lr}_{i j}}$, where $\operatorname{Lr}_{i j}$ is the maximum packet loss rate threshold of the path and $\mathrm{Hc}_{i j}$ is the maximum hop count threshold of the baseline path. $\overline{\operatorname{Lr}_{g k}}$ represents the fact that the packet loss rate threshold is averaged to every link in the path. The packet loss rate can be denoted by $\mathrm{lr}_{g k}=1-\left(\mathrm{pk}_{g k}^{e} / \mathrm{pk}_{g k}^{s}\right)$, where $\mathrm{pk}_{g k}^{e}$ is the total number of packets received by satellite node $k$ and $\mathrm{pk}_{g k}^{s}$ is the total number of packets sent by satellite node $g$. The smaller $\operatorname{lr}_{g k}$ is, the more consistent the optimal link. When $l r_{g k}$ is less than or equal to $\overline{\operatorname{Lr}_{g k}}, \mathrm{mf}_{g k}^{\mathrm{lr}}$ is invariably 1, which demonstrates that the current link completely conforms to the optimal link. When $\operatorname{lr}_{g k}$ is greater than $\overline{\operatorname{Lr}_{g k}}$, the membership degree of the packet loss rate decreases as $\operatorname{lr}_{g k}$ increases. That is, the degree of conformation to the optimal link decreases.

\subsubsection{Membership Degree Function of the Available} Bandwidth. Due to the high-speed movement of LEO satellites, the LEO satellite network is in a state of dynamic change, which results in the uncertainty of the available bandwidth. When $\mathrm{bd}_{g k}$ is less than $\overline{\mathrm{Bd}_{g k}}$, the degree of conformation of the available bandwidth to the optimal link increases rapidly at the beginning and then stabilizes relatively. When $b_{g k}$ is greater than or equal to $\overline{\mathrm{Bd}_{g k}}$, the available bandwidth is considered to have already conformed to the optimal link, so the membership degree function of the available bandwidth is invariably 1 . For that 
reason, the membership degree function of the available bandwidth of link $(g, k)$ is described by the logarithmic function as follows:

$$
\mathrm{mf}_{g k}^{\mathrm{bd}}= \begin{cases}1, & \mathrm{bd}_{g k} \geq \overline{\mathrm{Bd}_{g k}}, \\ \ln \left(\frac{\mathrm{bd}_{g k}}{\left.\overline{\mathrm{Bd}_{g k}}+e-1\right),}\right. & \mathrm{bd}_{g k}<\overline{\mathrm{Bd}_{g k}},\end{cases}
$$

where $\overline{\mathrm{Bd}_{g k}}$ is the available bandwidth threshold of link $(g, k)$. Since the available bandwidth is a concavity parameter, it is calculated as $\overline{\mathrm{Bd}_{g k}}=\left(\mathrm{bd}_{g k}^{\min }+\mathrm{bd}_{g k}^{\max }\right) / 2$, where $\mathrm{bd}_{g k}^{\mathrm{min}}$ is the minimum value of available bandwidth in the previous time period $t$ and $\mathrm{bd}_{g k}^{\max }$ is the maximum value of available bandwidth in the previous time period $t . \overline{\mathrm{Bd}_{g k}}$ represents the average available bandwidth threshold. The available bandwidth can be denoted by bd $\mathrm{d}_{g k}=\mathrm{bd}_{g k}^{\text {sum }}-\mathrm{bd}_{g k}^{\mathrm{oc}}$, where bd $\mathrm{b}_{g k}^{\mathrm{sum}}$ is the total bandwidth of link $(g, k)$ and bd $\mathrm{doc}^{\mathrm{oc}}$ is the occupied bandwidth of link $(g, k)$. The larger $b_{g k}$ is, the more consistent the optimal link. When $\mathrm{bd}_{g k}$ increases to $\overline{\mathrm{Bd}_{g k}}$, the membership degree of the available bandwidth increases. That is, the degree of conformation to the optimal link increases. When $\mathrm{bd}_{g k}$ is greater than $\overline{\mathrm{Bd}_{g k}}, \mathrm{mf}_{g k}^{\mathrm{bd}}$ is invariably 1 , which demonstrates that the current link completely conforms to the optimal link.

4.2. Comprehensive Evaluation of the Path. For the convenience of the routing calculation, ideal point theory [33] is employed to integrate these three link information criteria, i.e., the transmission delay, packet loss rate, and available bandwidth, into a comprehensive evaluation of the link. Euclidean distance is used to measure the distance between two points in ideal point theory. Therefore, Euclidean distance is adopted to calculate the distance between the membership degree function of the link information and the ideal point. This distance is defined as the comprehensive evaluation of the link. The comprehensive evaluation of the link from satellite node $g$ to satellite node $k$ is calculated as follows:

$$
\mathrm{ld}_{g k}=\sqrt{\left(\mathrm{mf}_{g k}^{\mathrm{td}}-1\right)^{2}+\left(\mathrm{mf}_{g k}^{\mathrm{lr}}-1\right)^{2}+\left(\mathrm{mf}_{g k}^{\mathrm{bd}}-1\right)^{2}} .
$$

The comprehensive evaluation of the path from the source satellite node $i$ to the destination satellite node $j$ can be obtained by accumulating the comprehensive evaluations of all links in path $_{i j}$ as follows:

$$
\operatorname{pd}_{i j}=\sum_{\forall(g, k) \in \text { path }_{i j}} \mathrm{ld}_{g k},
$$

where the smaller the $\mathrm{pd}_{i j}$ is, the greater the conformation of the path to the optimal path.

4.3. Routing Model Establishment. The routing model is established with the comprehensive evaluation of the path as the optimization objective, and the transmission delay of the path, the packet loss rate of the path, the available bandwidth of the path, and the hop count of the path as constraints. The LEO satellite network is regarded as a directed graph $G(V, E)$ using virtual nodes, where $V$ represents the set of all nodes and $E$ represents the set of all links in the satellite network. Suppose $i, j, g, k \in V,(g, k) \in E$, and path $_{i j}$ is the path from node $i$ to node $j$; then, the routing model of $G(V, E)$ is as follows:

$$
\begin{cases}\min & \left\{\mathrm{pd}_{i j}\right\} \\ & \sum^{\text {s.t. }} \mathrm{td}_{\mathrm{gk}} \leq \mathrm{Td}_{i j} \\ & \prod_{\forall(g, k) \in \text { path }_{i j}}\left(1-\mathrm{lr}_{\mathrm{gk}}\right) \geq 1-\mathrm{Lr}_{i j} \\ & \left\{\mathrm{bd}_{\mathrm{gk}}\right\} \geq \mathrm{Bd}_{i j} \\ \min _{\forall(g, k) \in \text { path }_{i j}} & \sum \mathrm{Hc}_{\mathrm{gk}} \leq \mathrm{Hc}_{i j} .\end{cases}
$$

In particular, $\mathrm{pd}_{i j}$ is the comprehensive evaluation of the path from the source satellite node $i$ to the destination satellite node j. $\sum_{\forall(g, k) \in \text { path }_{i j}} \mathrm{td}_{\mathrm{gk}} \leq \mathrm{Td}_{i j}$ is the transmission delay constraint of the path; that is, the sum of the transmission delays of all links in the selected path must be less than or equal to the maximum transmission delay threshold of path $\left(\mathrm{Td}_{i j}\right) . \prod_{\forall(g, k) \in \operatorname{path}_{i j}}\left(1-\operatorname{lr}_{\mathrm{gk}}\right) \geq 1-\operatorname{Lr}_{i j}$ is the packet loss rate constraint of the path; that is, the product of the reliability of every link in the selected path must be greater than or equal to the minimum reliability threshold of path $\left(1-\operatorname{Lr}_{i j}\right)$. In particular, $\operatorname{Lr}_{i j}$ is the maximum packet loss rate threshold of the path. $\min _{\forall(g, k) \in \text { path }_{i j}}\left\{\mathrm{bd}_{g k}\right\} \geq \mathrm{Bd}_{i j}$ is the available bandwidth constraint of the path; that is, the minimum available bandwidth of every link in the selected path must be greater than or equal to the minimum available bandwidth threshold of path $\left(\mathrm{Bd}_{i j}\right) . \sum_{\forall(g, k) \in \operatorname{path}_{i j}} H_{c_{g k}} \leq \mathrm{Hc}_{i j}$ is the hop count constraint of the path; that is, the hop count of the selected path must be less than or equal to the maximum hop count threshold of the baseline path. The baseline path is generated by a depth search with a depth limit $\left(\mathrm{Hc}_{i j}\right) . \quad \mathrm{Hc}_{i j}=$ num $_{\text {orbit }}+\left(\left(\right.\right.$ num $\left.\left._{\text {orbit_sat }}\right) / 2\right)$, where num $_{\text {orbit }}$ is the number of orbits of LEO satellite networks

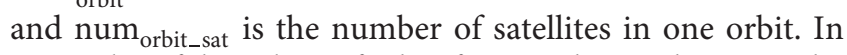
particular, if the values of $\mathrm{pd}_{i j}$ of two paths are the same, the optimal path is randomly selected from the two paths.

\section{Routing Model Solution Based on the Grey Wolf Optimization Algorithm}

The solution of the multiconstraint model is an NP-hard problem [34, 35]. The computational complexity of the routing model increases as the satellite network scale increases. As a new-element heuristic group intelligence algorithm, GWO was proposed by Mirjalili et al. [36]. GWO possesses the advantages of fast convergence and a small amount of required calculations, compared with traditional optimization algorithms. Due to the limited computing capacity of satellite networks, GWO is used to solve the routing model in this section. The flowchart of the solution of the routing model based on GWO is shown in Figure 2. 
The critical steps of GWO include individual position coding, initial population generation, fitness function design, and prey hunting.

5.1. Individual Position Coding. In this paper, the path is encoded by the satellite node sequence. An individual, which is recorded by a complete path from the source satellite node $i$ to the destination satellite node $j$, can be expressed as Paths $_{i j}=\{i, g, k, \ldots, m, j\}, i, g, k, m, j \in V$. Correspondingly, the path information set of path $_{i j}$ is expressed as $\mathrm{Info}_{i j}=\left\{\left(\mathrm{td}_{i g}, \mathrm{lr}_{i g}, \mathrm{bd}_{i g}\right),\left(\mathrm{td}_{g k}, \mathrm{lr}_{g k}, \mathrm{bd}_{g k}\right)\right.$

$\left., \ldots,\left(\mathrm{td}_{m j}, \mathrm{lr}_{m j}, \mathrm{bd}_{m j}\right)\right\}$. As shown in Figure 3, the link information of the source satellite node $i$ and the destination satellite node $j$ is, respectively, represented by $\left(\mathrm{td}_{i g}, \mathrm{lr}_{i g}, \mathrm{bd}_{i g}\right)$ and $\left(\mathrm{td}_{m j}, \mathrm{lr}_{m j}, \mathrm{bd}_{m j}\right)$, where the transmission delay, packet loss rate, and available bandwidth from satellite node ito satellite node $g$ are represented by $\mathrm{td}_{i g}, \mathrm{lr}_{i g}, \mathrm{bd}_{i g}$, respectively. In the process of solving the routing model, the source satellite node $i$ and the destination satellite node $j$ of each individual remain unchanged.
5.2. Initial Population Generation. To ensure the validity of the generated path, the initial path is randomly generated from the source satellite node $i$ to the destination satellite node $j$. Afterwards, the initial path is placed into the path set $\left(\right.$ Paths $_{i j}$ ).

5.3. Fitness Function Design. The optimization objective $\left(\mathrm{pd}_{i j}\right)$ of the routing model is used as the fitness function of GWO. The path set $\left(\right.$ Paths $_{i j}$ ) is sorted in ascending order according to the value of the fitness function, and then the top three individuals are selected as path $_{\alpha}$, path $_{\beta}$, and path in turn.

5.4. Prey Hunting. path $_{\alpha}$, path $_{\beta}$, and path $_{\delta}$ are considered as prey positions. Any path ${ }_{\omega}$ is continuously updated until the optimal path is obtained, as shown in Figure 4, and the updated path (path ${ }_{\omega}^{\prime}$ ) is calculated with the guidance of path $_{\alpha}$, path $_{\beta}$, and path $_{\delta}$ as follows:

$$
\begin{aligned}
R_{\alpha} & =\mid C_{1} \text { path }_{\alpha}-\text { path }_{\omega} \mid, \\
R_{\beta} & =\mid C_{2} \text { path }_{\beta}-\text { path }_{\omega} \mid, \\
R_{\delta} & =\mid C_{3} \text { path }_{\delta}-\text { path }_{\omega} \mid, \\
\operatorname{path}_{\omega}^{\prime} & =\frac{\text { path }_{\alpha}-A_{1} R_{\alpha}+\text { path }_{\beta}-A_{2} R_{\beta}+\text { path }_{\delta}-A_{3} R_{\delta}}{3},
\end{aligned}
$$

where $R_{\alpha}, R_{\beta}$, and $R_{\delta}$ are the absolute values of the relative distances between path ${ }_{\omega}$ and $\operatorname{path}_{\alpha}$, $\operatorname{path}_{\beta}$, and $\operatorname{path}_{\delta}$, respectively. $C_{1}, C_{2}$, and $C_{3}$ are the influence factors. $A_{1}, A_{2}$, and $A_{3}$ are the convergence impact factors.

\section{Simulation Analysis}

6.1. Simulation Environment. We simulate the proposed strategy in NS2 and Visual Studio to verify its effectiveness. The LEO satellite network is constructed with the Iridium constellation of polar orbits in this simulation. The network topology is composed of 66 LEO satellites, and the specific parameters are shown in Table 1 . The virtual node method is employed to solve the dynamic change issue of LEO satellite networks. According to the coverage area of each satellite in Iridium, Earth's surface is divided into 66 virtual nodes, which is equal to the number of LEO satellites. The positions and topologies of the 66 virtual nodes are shown in Figure 5.

Moreover, the routing update method is consistent with the literature [37]. A centralized routing computing method is adopted at the regional centre of the virtual nodes. LEO satellites monitor their link information in real time. When the monitored link information exceeds a certain threshold, the link information is sent to the regional centre of the virtual nodes. The route is calculated based on the collected link information at the regional centre. After the calculation is completed, the route is sent to the LEO satellites in the region.

A total of 120 user nodes are generated uniformly on the surface of Earth, and each user node generates traffic flows with the same probability. The source-destination pairs are randomly generated.

6.2. Simulation Results. The routing strategy for LEO satellite networks based on membership degree functions (RSSN-MDF) is compared with the routing strategy for satellite networks based on Dijkstra algorithm (RSSN-D), the routing strategy for satellite networks based on multilayer decision making (RSSN-ML) [38], and the routing strategy for satellite networks based on multiobjective decision making (RSSN-MDM) [39] in terms of average delay, delay jitter, packet loss rate, throughput, and comprehensive performance.

Figure 6 shows the comparison of the average delays at different packet sending rates. As shown in this figure, the average delay of RSSN-D increases rapidly as the packet sending rate increases. This is because RSSN-D only takes the transmission delay as the basis of the path calculation and easily becomes congested. RSSN-MDM takes the available bandwidth as one of the link evaluation indicators, effectively avoiding congestion. Consequently, the average delay of RSSN- 


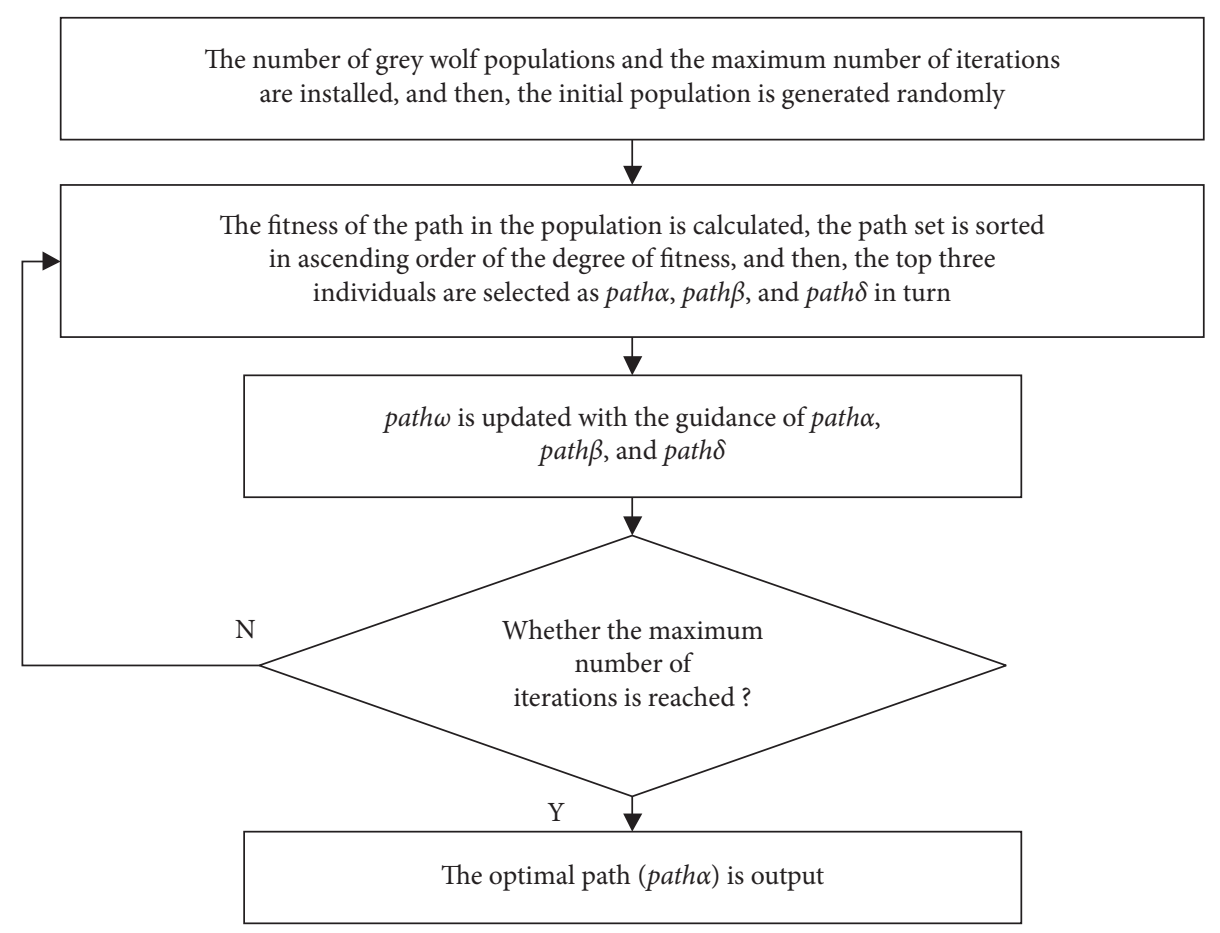

FIGURE 2: Flowchart of the solution of the routing model based on GWO.

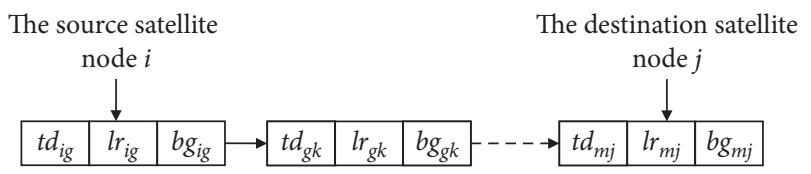

FIgURE 3: Individual position coding.

MDM is better than that of RSSN-ML. RSSN-MDF takes the transmission delay, packet loss rate, and available bandwidth as the optimization objectives and also considers the uncertainty of link information, so RSSN-MDF can adapt to complex space environments and avoid congestion to some extent. Thus, the optimal path can be obtained by RSSN-MDF. As a result, the average delay of RSSN-MDF is always low.

Figure 7 shows the comparison of delay jitters at different packet sending rates. The delay jitter is the difference between the maximum delay and the minimum delay when the packets pass through the path. As shown in this figure, the delay jitters of RSSN-MDM, RSSN-MDF, and RSSN-D are high and that of RSSN-ML is low. This is because RSSN-ML uses the hierarchical clustering method to calculate the route, which can reduce the complexity of the calculation and decrease the disturbance of the delay. RSSN-MDF takes the transmission delay of the path, packet loss rate of the path, available bandwidth of the path, and hop count of the path as constraints. Meanwhile, RSSN-MDF adopts a centralized routing computing method. The above reasons lead to higher computational complexity. Moreover, there is a delay in the routing update of RSSN-MDF. Some satellites may forward packets according to the old path, and some may forward packets according to the new path. Thus, the difference in the packet delays between RSSN-MDF and the other methods is large. As a result, the delay jitter of RSSN-MDF is high.

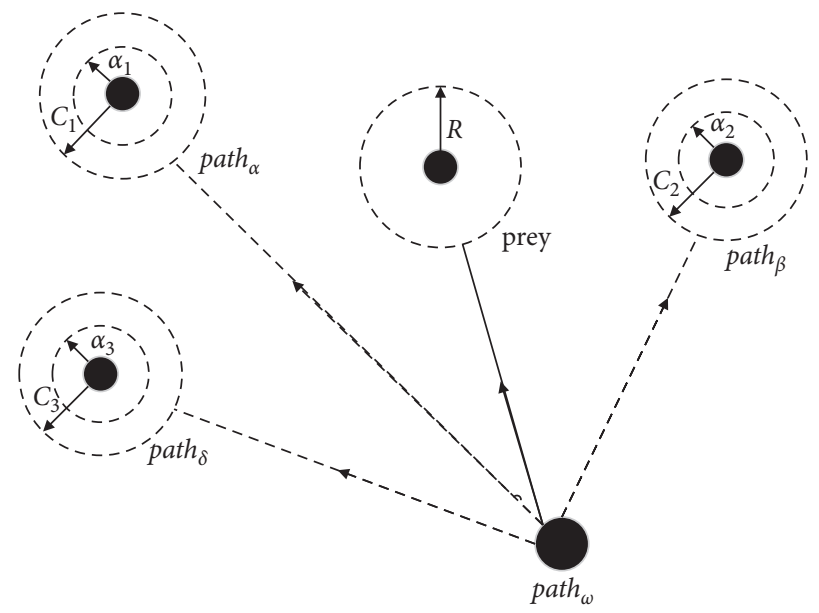

Figure 4: Prey hunting.

TABLE 1: Parameters of the LEO satellite network.

\begin{tabular}{lc}
\hline Parameters & Values \\
\hline Model & Iridium \\
Orbit number & 6 \\
Satellite number & 66 \\
Adjacent orbit interval & $27^{\circ}$ \\
Orbit height & $780 \mathrm{~km}$ \\
Link bandwidth & $25 \mathrm{mbps}$ \\
Queue buffer size & $50 \mathrm{~kb}$ \\
\hline
\end{tabular}

Figure 8 shows the comparison of packet loss rates at different packet sending rates. As shown in this figure, the packet loss rates of RSSN-MDF and RSSN-MDM are better 


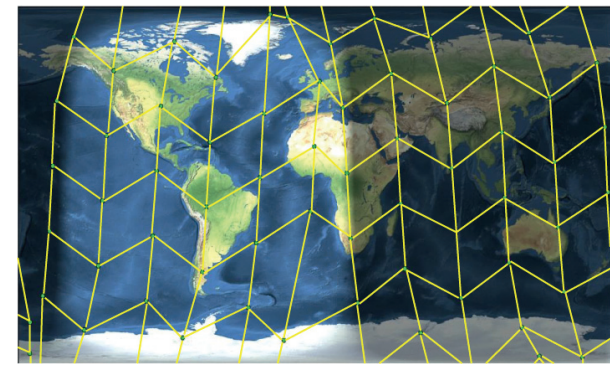

Figure 5: Positions and topologies of the virtual nodes.

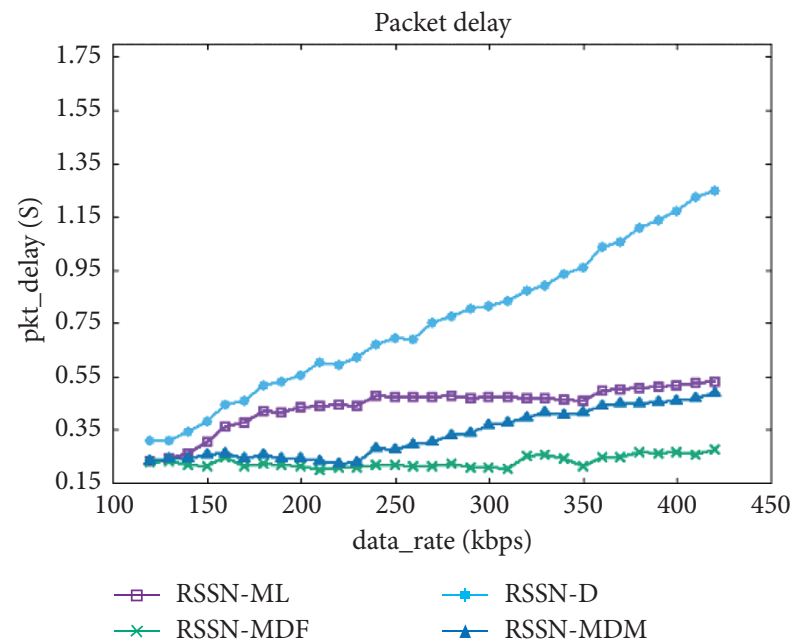

Figure 6: Comparison of average delays.

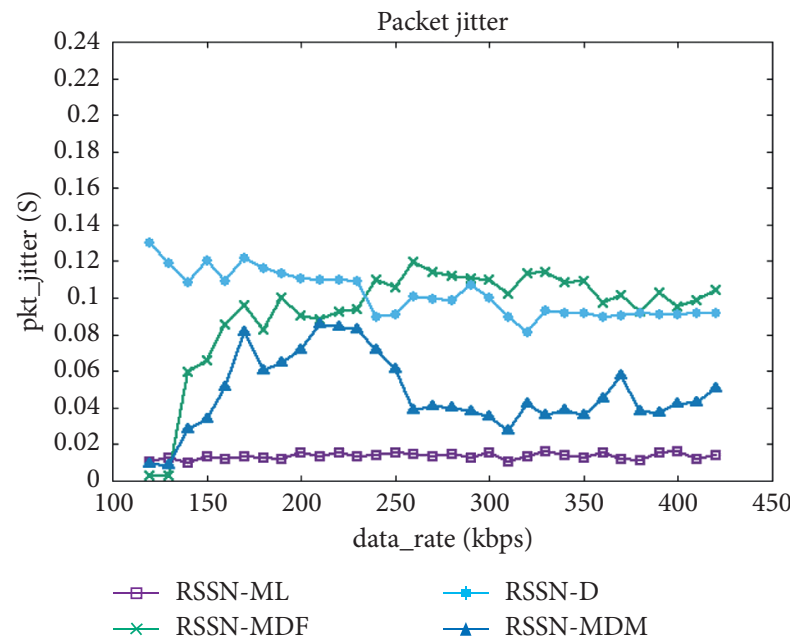

Figure 7: Comparison of delay jitters.

than those of RSSN-ML and RSSN-D. This is because RSSNMDF and RSSN-MDM are similar in terms of their transmission delays, packet loss rates, and available bandwidth as their link evaluation indicators. In addition, RSSN-ML and RSSN-D calculate their paths according to the determined values without considering the uncertainty of link information. Therefore, the packet loss rates of RSSN-ML and

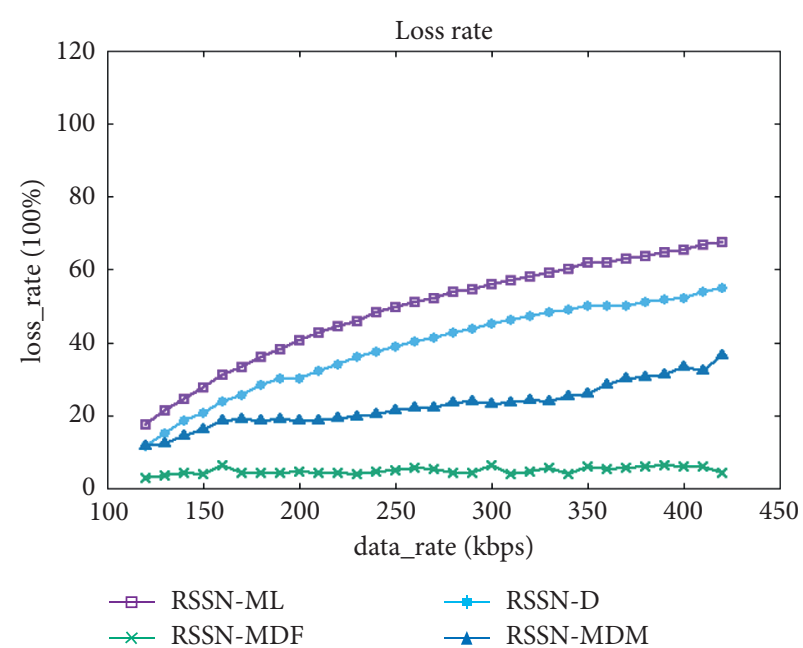

Figure 8: Comparison of packet loss rates.

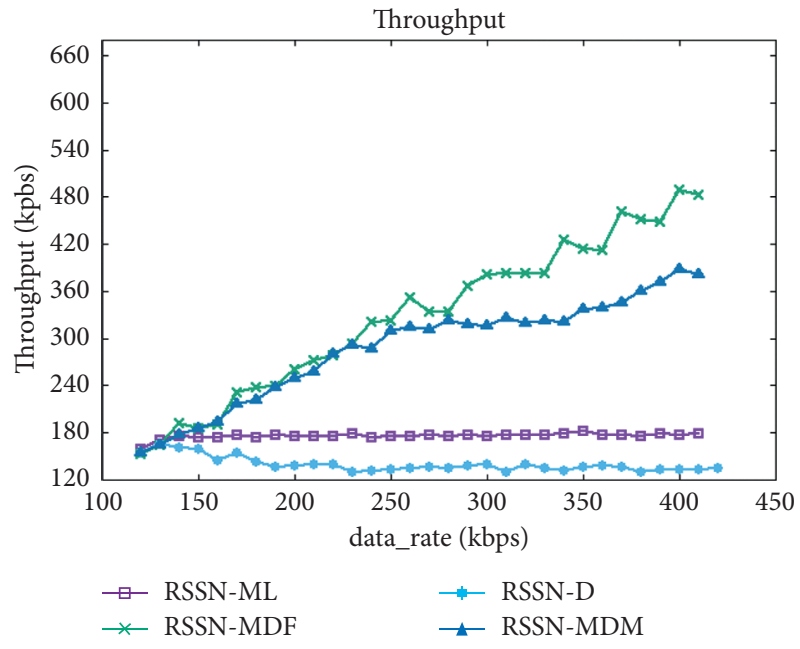

FIgURE 9: Comparison of throughputs.

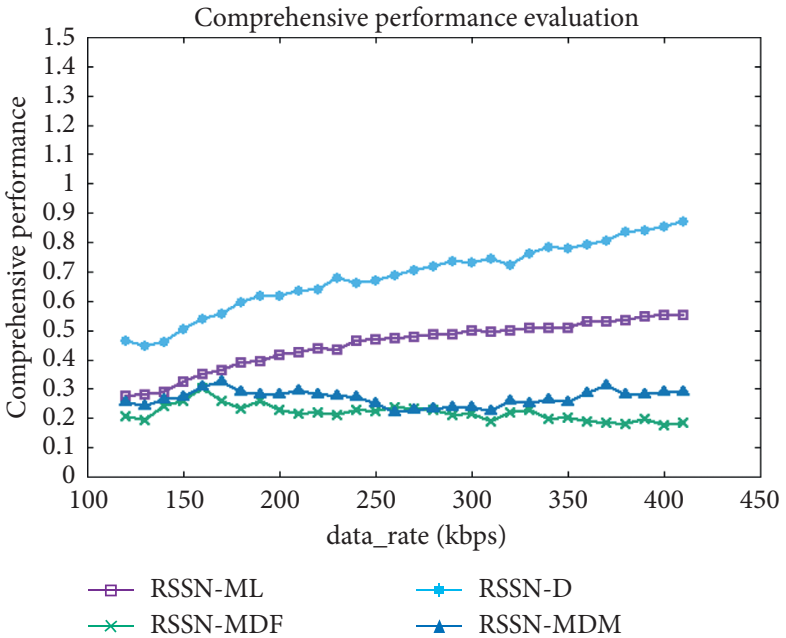

Figure 10: Comparison of comprehensive performances. 
RSSN-D rapidly increase as the packet sending rates increase. RSSN-MDF takes the uncertainty of link information into account and introduces path constraints into the routing model, which further improves the reliability of the routing calculation. As a result, the packet loss rate of RSSNMDF is always low.

Figure 9 shows the comparison of throughputs at different packet sending rates. As shown in this figure, the throughputs of RSSN-ML and RSSN-D are always low. This is because these two strategies calculate their paths according to the determined values; the optimal paths cannot be obtained in complex space environments. The throughput of RSSN-MDF is similar to that of RSSN-MDM at the beginning and then it becomes superior to that of RSSNMDM. This is because RSSN-MDF not only considers the uncertainty of link information but also takes the available bandwidth as the link evaluation indicator. Thus, RSSNMDF can adapt to complex space environments and avoid congestion to some extent. As a result, the throughput of RSSN-MDF increases as the packet sending rate increases.

To evaluate the overall performances of the four strategies, the comprehensive performance is used as an index. First, the average delay, delay jitter, packet loss rate, and throughput are all transformed into cost indexes, and minmax normalization is carried out. Then, the comprehensive performance is calculated by the average weighted summation of these normalized indexes. The smaller the comprehensive performance value is, the better the routing strategy will perform. Figure 10 shows the comparison of comprehensive performances at different packet sending rates. As the packet sending rates increase, the comprehensive performances of RSSN-D and RSSN-ML worsen, while those of RSSN-D and RSSN-ML are relatively stable. As shown in this figure, the comprehensive performance of the proposed strategy is the best. In summary, RSSN-MDF can achieve efficient and secure routing in complex space environments.

\section{Conclusions}

Research on routing strategies is significant for MEC satellites. However, the complexity of LEO satellite networks leads to the uncertainty of link information. In this paper, a routing strategy for LEO satellite networks based on membership degree functions is proposed. First, a routing model based on uncertain link information is established. Specifically, the uncertain link information is described by the designed membership degree function, and then the comprehensive evaluation of the path is obtained by integrating different link information of each link in the path. Afterwards, the comprehensive evaluation of the path is taken as the optimization objective to establish the routing model. Second, a GWO algorithm is designed to solve the routing model. Finally, simulation results show that the proposed strategy can achieve efficient and secure routing in complex space environments. Although the proposed strategy loses some delay jitter, it improves the performance in terms of average delay, packet loss rate, and throughput, compared with the performances of traditional routing strategies. The influence of the degree of uncertainty will be taken into consideration in future research.

\section{Data Availability}

The simulated evaluation data used to support the findings of this study are available from the corresponding author upon request.

\section{Conflicts of Interest}

The authors declare that there are no conflicts of interest regarding the publication of this paper.

\section{Acknowledgments}

This work was supported by the National Natural Science Foundation of China under Grants 61972210, 61873131, 61872191, and 61803212 and by the Natural Science Foundation of the Higher Education Institutions of Jiangsu Province under Grant 18KJB520034.

\section{References}

[1] X. Xu, Q. Wu, L. Qi, W. Dou, S.-B. Tsai, and M. Z. A. Bhuiyan, "Trust-Aware service offloading for video surveillance in edge computing enabled internet of vehicles," IEEE Transactions on Intelligent Transportation Systems, vol. 14, p. 1, 2020.

[2] W. Hou, Z. Ning, and L. Guo, "Green survivable collaborative edge computing in smart cities," IEEE Transactions on Industrial Informatics, vol. 14, no. 4, pp. 1594-1605, 2018.

[3] X. Xu, X. Liu, Z. Xu, F. Dai, X. Zhang, and L. Qi, "Trustoriented IOT service placement for smart cities in edge computing," IEEE Internet of Things Journal, vol. 7, no. 5, pp. 4084-4091, 2020.

[4] Y. Lu, Y. J. Zhao, F. C. Sun et al., "Routing techniques on satellite networks," Journal of Software, vol. 25, no. 5, pp. 1085-1100, 2014.

[5] J. Sun, Y. Zhang, Z. Wu et al., "An efficient and scalable framework for processing remotely sensed big data in cloud computing environments," IEEE Transactions on Geoscience and Remote Sensing, vol. 57, no. 7, pp. 4294-4308, 2019.

[6] Z. Zhang, W. Zhang, and F. H. Tseng, "Satellite mobile edge computing: improving QoS of high-speed satellite-terrestrial networks using edge computing techniques," IEEE Network, vol. 33, no. 1, pp. 70-76, 2018.

[7] F. Wang, D. Jiang, S. Qi, C. Qiao, and L. Shi, "A dynamic resource scheduling scheme in edge computing satellite networks," Mobile Networks and Applications, vol. 14, 2020.

[8] L. Yan, S. Cao, Y. Gong et al., "SatEC: a 5G satellite edge computing framework based on microservice architecture," Sensors, vol. 19, no. 4, Article ID 831, 2019.

[9] Y. Wang, J. Yang, X. Guo, and Z. Qu, "A game-theoretic approach to computation offloading in satellite edge computing," IEEE Access, vol. 8, pp. 12510-12520, 2020.

[10] Y. Wang, J. Yang, X. Guo et al., "Satellite edge computing for the internet of things in aerospace," Sensors, vol. 19, no. 20, Article ID 4375, 2019.

[11] J. Wei, J. Han, and S. Cao, "Satellite IoT edge intelligent computing: a research on architecture," Electronics, vol. 8, no. 11, Article ID 1247, 2019.

[12] P. Cao, M. M. Fan, K. Liu et al., "Dynamic programming modeling of satellite system communication routing 
problems," in Proceedings of the National Youth Conference on Information and Management Sciences, New York, NY, USA, 2012.

[13] L. Wood, A. Clerget, I. Andrikopoulos et al., "IP routing issues in satellite constellation networks," International Journal of Satellite Communications, vol. 19, pp. 69-92, 2010.

[14] M. Werner, "A dynamic routing concept for ATM-based satellite personal communication networks," IEEE Journal on Selected Areas in Communications, vol. 15, no. 8, pp. 16361648, 1997.

[15] D. Fischer, D. Basin, and T. Engel, "Topology dynamics and routing for predictable mobile networks," in Proceedings of the IEEE International Conference on Network Protocols, Berlin, Germany, 2008.

[16] J. Wang, L. Li, and M. Zhou, "Topological dynamics characterization for LEO satellite networks," Computer Networks, vol. 51, no. 1, pp. 43-53, 2007.

[17] R. Mauger and C. Rosenberg, "QoS guarantees for multimedia services on a TDMA-based satellite network," IEEE Communications Magazine, vol. 35, no. 7, pp. 56-65, 1997.

[18] E. Ekici, I. F. Akyildiz, and M. D. Bender, "A distributed routing algorithm for datagram traffic in LEO satellite networks," IEEE/ACM Transactions on Networking, vol. 9, no. 2, pp. 137-147, 2001.

[19] Y. Hashimoto, "Design of IP-based routing in a LEO satellite network," in Proceedings of the International Workshop on Satellite-Based Information Services, London, UK, 1998.

[20] H. Nishiyama, Y. Tada, N. Kato et al., "Toward optimized traffic distribution for efficient network capacity utilization in two-layered satellite networks," IEEE Transactions On Vehicular Technology, vol. 62, pp. 1303-1313, 2012.

[21] F. Xiao, L. J. Sun, X. G. Ye et al., "Routing algorithm for MPLS traffic engineering in satellite network," Journal on Communications, vol. 35, pp. 104-111, 2011.

[22] J. Wang, Y. J. Guo, L. J. Sun et al., "Load balancing algorithm for multi-traffic in double layered satellite network," Journal of Systems Engineering and Electronics, vol. 38, pp. 2156-2161, 2016.

[23] J. Feng and G. Q. Gu, "Research on QoS routing based on uncertain parameters," Journal of Computer Research and Development, vol. 39, pp. 553-539, 2002.

[24] X.-W. Wang, Q. Wang, M. Huang et al., "A fuzzy integral and game theory based QoS multicast routing scheme," Journal of Software, vol. 19, no. 7, pp. 1743-1752, 2008.

[25] L. Cobo, A. Quintero, and S. Pierre, "Ant-based routing for wireless multimedia sensor networks using multiple QoS metrics," Computer Networks, vol. 54, no. 17, pp. 2991-3010, 2010.

[26] X.-M. Wang, J.-L. Lu, Y.-S. Li, and K.-G. Hao, "Multiconstrained multipath routing for wireless sensor networks in the fuzzy random environment," Chinese Journal of Computers, vol. 34, no. 5, pp. 779-791, 2011.

[27] X. Y. Zhang and T. Zhang, "The mechanism based on fuzzy integral in multi-attribute optimal routing," in Proceedings of the IEEE International Symposium on Microwave, Antenna, Propagation and EMC Technologies for Wireless Communications, New York, NY, USA, 2011.

[28] C. Li, C. Liu, Z. Jiang et al., "A novel routing strategy based on fuzzy theory for NGEO satellite networks," in Proceedings of the IEEE Vehicular Technology Conference, Berlin, Germany, 2015.

[29] Z. Jiang, C. Liu, S. He et al., "A QoS routing strategy using fuzzy logic for NGEO satellite IP networks," Wireless Networks, vol. 24, pp. 1-13, 2018.
[30] X. Zhou, G. Zhang, J. Sun, J. Zhou, T. Wei, and S. Hu, "Minimizing cost and makespan for workflow scheduling in cloud using fuzzy dominance sort based HEFT," Future Generation Computer Systems, vol. 93, pp. 278-289, 2019.

[31] X. Y. Zhou, K. Q. Zou, and Y. F. Wang, "Fuzzy variable time series based on fuzzy membership function and econometrics," in Proceedings of the International Symposium on Knowledge Acquisition and Modeling, New York, NY, USA, 2010.

[32] S. L. Chen, J. G. Li, and X. G. Wang, Fuzzy Set Theory and its Application, Science Press, Beijing, China, 2005.

[33] C. L. Hwang and K. Yoon, Multiple Attribute Decision Making, Spring-Verlag, Berlin, Germany, 1981.

[34] M. Miklós, B. Alia, and L. Samer, "The cost optimal solution of the multi-constrained multicast routing problem," Computer Networks, vol. 56, pp. 3136-3149, 2012.

[35] J. L. Zhou, J. Sun, P. J. Cong et al., "Security-critical energyaware task scheduling for heterogeneous real-time MPSoCs in IoT," IEEE Transactions on Services Computing, vol. 13, no. 4, pp. 1-14, 2020.

[36] S. Mirjalili, S. M. Mirjalili, and A. Lewis, "Grey wolf optimizer," Advances in Engineering Software, vol. 69, pp. 46-61, 2014.

[37] S. D. Zhang, L. J. Sun, J. Zhou et al., "Destruction-resistant routing strategy for GEO/LEO double-layer satellite networks," Journal of Nanjing University of Posts and Telecommunications, vol. 38, pp. 1-7, 2018.

[38] I. F. Akyildiz, E. Ekici, and M. D. Bender, "MLSR: a novel routing algorithm for multilayered satellite IP networks," IEEE/ACM Transactions on Networking, vol. 10, no. 3, pp. 411-424, 2002.

[39] L. Yang, J. Sun, C. S. Pan et al., "LEO multi-service routing algorithm based on multi-objective decision making," Journal on Communications, vol. 37, pp. 25-32, 2016. 